\title{
Brief group-based acceptance and commitment therapy for stroke survivors
}

\author{
Sarah Majumdar and Reg Morris* (iD \\ South Wales Doctoral Programme in Clinical Psychology, Cardiff University, UK
}

Objectives. To date, the efficacy of acceptance and commitment therapy (ACT) for stroke survivors has not been established. The aim of this study was to evaluate the efficacy of groupbased ACT for stroke survivors in comparison with treatment as usual (TAU) controls.

Methods. Fifty-three participants were randomly assigned either to group-based ACT (ACTivate Your Life after Stroke) or to a TAU control group (60\% male; mean age: 63 years). The ACT intervention consisted of four weekly 2-hr didactic group sessions. Therapeutic effects were measured by examining changes in depression (primary outcome), anxiety, hope, health-related quality of life, self-rated health status, and mental well-being. Measures were completed at pre-treatment, post-treatment, and 2-month follow-up. A mixed-design repeated-measures multivariate ANOVA was conducted to analyse the findings.

Results. Analysis based on intention to treat found that compared to participants in the TAU control, group-based ACT significantly reduced depression and increased self-rated health status and hopefulness in stroke survivors, with medium effect sizes. Significantly more participants reached clinically significant change of depression in the ACT intervention in comparison with the control group.

Conclusions. The results correspond with previous studies of group-based ACT with other long-term conditions. The findings from this current study suggest group-based ACT may have promising utility and could offer a suitable low-intensity psychological intervention for stroke survivors. However, further large-scale research is required.

\section{Practitioner points}

- Acceptance and commitment therapy (ACT), delivered didactically to groups of stroke survivors, proved feasible and acceptable.

- ACT had benefits, relative to treatment as usual, for depression, health status, and hope.

- Several secondary outcome variables did not show dependable benefit for ACT: anxiety; healthrelated quality of life; and mental well-being.

- Results should be treated as preliminary as the sample size was small, blinding was not possible, concomitant treatments were not monitored, and there was no attention control condition.

- Despite these limitations, group-based ACT merits further study as a potentially effective intervention. This is an open access article under the terms of the Creative Commons Attribution-NonCommercial-NoDerivs License, which
permits use and distribution in any medium, provided the original work is properly cited, the use is non-commercial and no
modifications or adaptations are made.

*Correspondence should be addressed to Reg Morris, Cardiff University, 70 Park Place, Tower building, I I th floor, Cardiff CFI 0 3AT, UK (email: Reg.Morris@wales.nhs.uk). 
Stroke is defined as 'rapidly developing clinical signs of focal (or global) disturbance of cerebral function, with symptoms lasting 24 hrs or longer, or leading to death' (WHO Monica Project Principal Investigators, 1988). Approximately 17 million strokes occur every year resulting in 5.9 million deaths and 33 million stroke survivors living worldwide (Feigin et al., 2014). Stroke affects many physical functions, and consequently, $40 \%$ of stroke survivors are discharged from hospital requiring support with activities of daily living (Royal College of Physicians, 2015). Stroke is considered to be one of the most common causes of complex disability, affecting over half of all stroke survivors (Adamson, Beswick, \& Ebrahim, 2004). The worldwide burden of stroke-related disability, illness, and premature death is set to double by 2030 (Feigin et al., 2014).

Psychological disabilities are common (Stroke Association, 2013). The prevalence of post-stroke depression is 31-33\% (Hackett \& Pickles, 2014) with 55\% of stroke survivors experiencing depression in the 15 years after stroke (Ayerbe, Ayis, Wolfe, \& Rudd, 2013; Hackett, Yapa, Parag, \& Anderson, 2005). The incidence of anxiety up to 10 years poststroke ranges from $17 \%$ to $24 \%$, with $57 \%$ experiencing anxiety during this period (Ayerbe, Ayis, Crichton, Wolfe, \& Rudd, 2014). Moderate and severe fatigue affects $57 \%$ of stroke survivors (Lerdal et al., 2011). The Stroke Association (2016) found that $73 \%$ of stroke survivors lacked confidence, $56 \%$ felt people treated them differently, and $55 \%$ felt unable to care for their families as before. Stroke-related comorbidities are associated with reduced quality of life (Bays, 2001; Godwin, Ostwald, Cron, \& Wasserman, 2013; Sturm et al., 2004), increased mortality (Ayerbe et al., 2013), increased health care utilization (Appleby, Thompson, \& Galea, 2012; Ghose, Williams, \& Swindle, 2005; van Eeden et al., 2016), and reduced functional recovery (Gillen, Tennen, McKee, \& Gernert-Dott, 2001).

\section{Psychological interventions for stroke survivors}

There is a lack of clarity regarding effective psychological interventions for stroke survivors (Royal College of Physicians, 2016). A randomized controlled trial (RCT) found cognitive behavioural therapy (CBT) to be ineffective with stroke survivors and reported that cognitive strategies were challenging for participants to implement (Lincoln \& Flannaghan, 2003). Several systematic reviews have concluded that there is limited evidence for the efficacy of psychosocial therapies including problem-solving, goal setting, psychoeducation, and social support (Hackett, Anderson, House, \& Halteh, 2008; Hackett, Anderson, House, \& Xia, 2008; Sugavanam, Mead, Bulley, Donaghy, \& van Wijck, 2013). Moreover, condition-specific reviews have concluded that there is only weak evidence for efficacy of treatments for several common post-stroke conditions: depression (Hackett, Anderson, House, \& Xia, 2008); anxiety (Knapp et al., 2017); fatigue (Wu et al., 2015); executive functioning (Chung, Pollock, Campbell, Durward, \& Hagen, 2013); and cognitive function (Gillespie et al., 2015; Loetscher \& Lincoln, 2013).

The UK National Clinical Guidelines for Stroke (Royal College of Physicians, 2016) propose brief psychological interventions for all stroke survivors with, or at risk of, depression or anxiety. Recommended interventions include motivational interviewing (MI), problem-solving therapy, or behavioural therapy (adapted cognitive behaviour therapy) (Royal College of Physicians, 2016). An RCT reported that MI, delivered 1:1 over four sessions, showed significant effects on mood at 3 months after stroke in comparison with a TAU group (Watkins et al., 2007) and improved mood and reduced mortality at 12 months (Watkins et al., 2011). Behavioural therapy, compared with TAU in an RCT, produced significant improvements in self-reported and observer-rated mood and selfesteem at 3 months in stroke survivors with aphasia and low mood (Thomas, Walker, 
Macniven, Haworth, \& Lincoln, 2013). Cost analysis showed some savings in resource utilization at 6-month follow-up in comparison with a TAU group (Humphreys, Thomas, Phillips, \& Lincoln, 2014). Group-based problem-solving therapy significantly improved task-oriented coping but not disease-specific quality of life in stroke survivors at 6 months in comparison with a TAU control (Visser et al., 2015). Despite these few encouraging findings, the psychological interventions required resource-intensive delivery formats utilizing 1:1 therapy or numerous group session. Therefore, there are limited intervention options for stroke survivors; yet almost three-quarters of stroke survivors experiencing emotional distress felt their psychological needs were not being met (McKevitt et al., 2011). This potentially hampers recovery (Gillen et al., 2001) and adds to the health service resource burden produced by long-term conditions (Department of Health, 2012), thus highlighting the pressing need to evaluate a greater range of therapeutic approaches for stroke (Kangas \& McDonald, 2011; Soo, Tate, \& Lane-Brown, 2011) with a focus on cost-effective delivery formats, such as group-based interventions (Hunsley, 2002).

\section{Acceptance and commitment therapy}

Acceptance and commitment therapy (ACT) proposes that psychological distress is a natural aspect of human experience. Its primary aim is to promote acceptance and not to rid a person of their distress. ACT aims to increase 'psychological flexibility' which allows pursuit of a valued life despite physical limitations, painful thoughts, feelings, or sensations (Harris, 2013). There is a growing body of research in support of ACT across a wide range of clinical populations (Graham, Gouick, Krahé, \& Gillanders, 2016; Ost., 2014; Ruiz, 2012; Swain, Hancock, Dixon, Koo, \& Bowman, 2013; Veehof, Trompetter, Bohlmeijer, \& Schreurs, 2016). Group-based ACT has been applied to several health conditions including cancer, multiple sclerosis, epilepsy, diabetes, and chronic pain with promising findings in reducing depression, anxiety, and disability and increasing acceptance and other condition-specific outcomes (Gregg, Callaghan, Hayes, \& GlennLawson, 2007; Kemani et al., 2015; Lundgren, Dahl, Melin, \& Kies, 2006; Lundgren, Dahl, Yardi, \& Melin, 2008; McCracken, Sato, \& Taylor, 2013; Mohabbat-Bahar, Maleki-Rizi, Akbari, \& Moradi-Joo, 2015; Nordin \& Rorsman, 2012; Wetherell et al., 2011). Moreover, a single case report found benefits for ACT with an adult experiencing post-stroke anxiety (Graham, Gillanders, Stuart, \& Gouick, 2014). ACT has a number of characteristics that suggest its suitability for stroke:

- It adopts a health model rather than an illness model (Hayes, Strosahl, \& Wilson, 1999) and is conducive to the promotion of well-being rather than simply the removal of symptoms.

- Its emphasis on acceptance of distress and 'getting on with life' is suited to stroke because, in many people, physical effects such as hemiplegia are enduring and even psychological effects such as anxiety may persist for many years (Astrom, 1996).

- ACT seeks to change the functions of cognitions about a person's condition by teaching them to distinguish between a thought and the event itself. It does not expect thoughts to be altered or reduced in frequency (like traditional CBT) - something that may be difficult for a person with a major, persistent condition such as aphasia or hemiplegia.

- The inclusion of mindfulness and 'being in the present' encourages a person to make contact with their surroundings and to be open to experiences beyond their disability and distress.

- ACT promotes the sense of self ('self-as-context') which helps build a sense of an enduring and observing self that is separate from the experience of distress and life's 
tribulations. This emphasis on self-as-context is potentially helpful with detrimental change in self-identity after stroke (Lapadatu \& Morris, 2017).

- The discovery of a person's core values and the facilitation of their use in value-based goal setting and 'committed action' are key features of ACT that may represent an improvement on the traditional goal setting practice in stroke which has been criticized (Sugavanam et al., 2013; Plant, Tyson, Kirk, \& Parsons, 2016).

\section{Objectives of current study}

The aim was to assess the efficacy of brief group-based ACT for stroke survivors ('ACTivate Your Life after Stroke') in comparison with a treatment as usual (TAU) control group using random allocation to conditions.

Non-acceptance of the psychological effects of stroke-related disability is associated with depression after controlling for age, gender, original stroke severity, and current disability at 1- and 9-month follow-ups (Townend, Tinson, Kwan, \& Sharpe, 2010). Therefore, it was hypothesized that stroke survivors who attend the ACT intervention will exhibit a greater reduction in depression (primary outcome) across pre-treatment, posttreatment, and follow-up time points, in comparison with the control group. It was also hypothesized that similar benefits of ACT would be observed for anxiety, health-related quality of life (HRQoL), self-reported health status, hopefulness, ${ }^{1}$ and mental well-being (secondary outcomes).

\section{Methods}

\section{Participants and procedure}

This study was advertised to members of the stroke clinical teams across three NHS sites in South Wales and one in south west England. Leaflets detailing the course content and aims ('a short course to help you get on with your life after stroke') were distributed to the clinicians to pass on to their patients who met the inclusion criteria of at least one clinically diagnosed stroke, were discharged from hospital, were over 18 years old, and did not have severe communication difficulties (e.g., aphasia) or cognitive impairments. Stroke survivors were not eligible if they had another acquired brain injury (e.g., traumatic brain injury, encephalitis, tumours), a diagnosed degenerative condition (e.g., dementia), or a severe mental illness (e.g., psychosis).

Internet software (www.randomizer.org) generates a random number sequence to determine group allocation, and a parallel group study design was utilized. Participants interested in this study were referred by members of the clinical team to a designated person within each site (not the researcher), who was responsible for enrolling participants and group allocation. Once participants consented (in ignorance of the next assignment in the sequence), they were consecutively randomized to conditions. No restrictions to randomization were used. Letters were then sent to participants indicating which group they had been allocated to and their course dates. For obvious reasons, this study could not be blinded. All outcome measures were collected by the researcher at pre-intervention (immediately prior to the first session), post-intervention (immediately following the final session), and at 2-month follow-up, which were

\footnotetext{
' Hopefulness was selected as a process measure in preference to the Acceptance and Action Questionnaire (AAQ; Bond et al., 2004) as a non-randomized pilot study (Ivey-Williams, 20I5) indicated that the AAQ may not be suitable for stroke patients.
} 
distributed by post. The control group responded at the same time points, returning responses by post or over the telephone. Prepaid self-addressed envelopes were provided for postal returns. No monetary incentives were used to recruit or retain participants.

\section{Study conditions}

\section{ACT intervention}

The ACT intervention, 'ACTivate Your Life after Stroke', consisted of 2-hr weekly didactic PowerPoint group sessions, for four consecutive weeks. The intervention is described in more detail in Cartwright and Hooper (2017). In sessions one and four, an extra half an hour was allocated for study questionnaire completion. Due to the trans-diagnostic nature of ACT (Lang et al., 2012), carers were invited to the course but were not part of the study analysis. As the intervention was didactic and non-interactive, the presence of carers was not expected to inhibit the survivors, but it may have enhanced confidence and sense of support.

The intervention was developed from Professor Neil Frude's 'ACTivate your life' course, which has been employed across NHS mental health services within South Wales, UK (Cartwright \& Hooper, 2017), and has received positive appraisal from Steven Hayes, co-founder of ACT (S. C. Hayes, personal communication to Prof N. Frude, 21/12/2014). The material was manualized and psychoeducational in nature, delivered by Microsoft PowerPoint with several ACT-based individual activities throughout, such as guided mindfulness practices. The mental health version of the course was adapted for stroke in collaboration with stroke survivors and carers. Changes included reducing contrasting colours, simplifying the language and number of words on the slides, and inclusion of stroke specific examples. The modified version was used across all four sites. The session-by-session outline is illustrated in Table 1. A handout was provided for each session which included a session summary and suggested home activities.

Courses were run in community venues, for example, libraries across four sites (three NHS in south Wales and one-third sector organization in south west England). Sessions had at least two facilitators consisting of clinical psychologists, assistant psychologists, or stroke care co-ordinators. There was at least one clinical psychologist within the presenting team at each site. To ensure fidelity, all of the course facilitators received the same intensive 2 day training facilitated by Prof. Neil Frude and received clinical supervision. The didactic nature of the intervention, in which the material was presented on slides and read by the facilitators, further ensured fidelity.

\section{TAU control group}

Participants in the control group followed their usual treatments. This meant that they had access, should they choose, to community services that are available to all, for example, GP, charity support, or online resources. At the time of this study, there were no dedicated local NHS community stroke services for stroke survivors with psychological difficulties beyond 6 weeks after discharge. The control group were offered the ACT intervention, after the 2-month follow-up, which consisted of the exact same material, course facilitators, and time period as the first group. 
Table I. Session-by-session outline of 'ACTivate Your Life after Stroke' course

\begin{tabular}{|c|c|}
\hline Session & Content \\
\hline Week one: You are not your mind & $\begin{array}{l}\text { Creating the distinction between actions that are under our own } \\
\text { (conscious, deliberate) control and actions that are controlled by } \\
\text { our mind (e.g., on 'autopilot') including experiences of self- } \\
\text { criticism and rumination. Introduced the idea of developing a } \\
\text { viewpoint from which one can observe thoughts and feelings, non- } \\
\text { judgementally }\end{array}$ \\
\hline Week two: Facing up to life & $\begin{array}{l}\text { Focus on acceptance (not resignation) and willingness to experience } \\
\text { unpleasant feelings and sensations (e.g., pain or anxiety) without } \\
\text { attempts to fight, avoid or suppress them, which can causes long- } \\
\text { term suffering when this gets in the way of meaningful activity }\end{array}$ \\
\hline Week three: Being mindful & $\begin{array}{l}\text { Thoughts are thoughts, not facts. Several examples of thought } \\
\text { defusion exercises presented. Explanation of mindfulness and non- } \\
\text { interactive activities completed (e.g., body scan) }\end{array}$ \\
\hline Week four: Living wisely, living well & $\begin{array}{l}\text { Identification of individual values, distinction between values and } \\
\text { goals and examples of committed action discussed }\end{array}$ \\
\hline
\end{tabular}

\section{Measures}

Socio-demographical information

Information was collected regarding: age, gender, date of first stroke and most recent stroke (if applicable), type and location of stroke (if known), employment status, living arrangements, and experience of mental health conditions since stroke and therapy received, if applicable.

\section{Primary measure}

\section{Depression}

Depression was chosen as the primary outcome as it is widely used as such in stroke research and had a strong association with recovery (Ayerbe et al., 2013) as well as a range of other key outcomes such as quality of life, increased use of health services, and greater risk of hospitalization and institutionalization (Lincoln, Kneebone, Macniven, \& Morris, 2012). It has also been found to be associated with the important ACT process of acceptance of disability after stroke (Townend et al., 2010). The Patient Health Questionnaire-9 (PHQ-9) measure was used to screen for depression and includes items about affect, behaviour, and somatic symptoms. It is widely used and had good validity (sensitivity of $88 \%$ and specificity of $88 \%$ ), internal reliability (Cronbach's $\alpha$ of .89), and test-retest reliability (correlation $=.84$ ) in a primary care sample (Kroenke, Spitzer, \& Williams, 2001) and performs well as a screening tool for post-stroke depression (Williams et al., 2005).

\section{Secondary measures}

\section{Anxiety}

The Generalized Anxiety Disorder-7 measure was used to screen for anxiety (Spitzer, Kroenke, Williams, \& Lowe, 2006). It had good validity (sensitivity of $89 \%$ and specificity of $82 \%$ ), internal reliability (Cronbach's $\alpha$ of .92), and test-retest reliability (intraclass 
correlation; ICC $=.83$ ) in a primary care sample. It is also moderately good at screening for a variety of other anxiety disorders (Kroenke, Spitzer, Williams, Monahan, \& Lowe, 2007).

\section{Health-related quality of life}

The EQ-5D-5L (Herdman et al., 2011) had good internal reliability (ICC $=.57$ ) and testretest reliability (ICC $=.69$ ) across health samples (Janssen, Birnie, Haagsma, \& Bonsel, 2008) and has been validated in stroke samples (Dorman, Waddell, Slattery, Dennis, \& Sandercock, 1997; Golicki et al., 2015). Scores from this current study were converted to index value sets as recommended by the EuroQol Group as these index value sets reflect the health state of the general population in any given country (Devlin, Shah, Feng, Mulhern, \& van Hout, 2018).

\section{Self-reported health status}

Part two of the EQ-5D-5L (Herdman et al., 2011) was used to measure self-reported health status. This consists of a $20 \mathrm{~cm}$ visual analogue scale which asks users to indicate from 0 to 100 'how good is your health today?' Test-retest reliability was reported as ICC $=.51$ across health samples (Janssen et al., 2008) and has been validated in stroke samples (Dorman et al., 1997; Golicki et al., 2015).

\section{Hope}

The Adult Hope Scale (Snyder et al., 1991) was used to assess hope. Total scores from the eight active items range from 0 to 48 , with higher scores indicating greater hopefulness. The questionnaire was internally consistent (Cronbach's $\alpha$ ranging from .74 to .84); showed good test-retest reliability (Cronbach's $\alpha$ ranging from .73 to .85 ) and good validity across student and clinical populations (Snyder, 2002; Snyder et al., 1991).

\section{Mental well-being}

Warwick and Edinburgh Mental Well-being Scale had good validity, internal consistency (Cronbach's $\alpha=.91)$, and test-retest reliability $(\mathrm{ICC}=.83$ ) in general population and student samples (Tennant et al., 2007). It comprises of 14 items, and a higher score indicates greater mental well-being. Mental well-being describes positive states of being, thinking, behaving, and feeling.

\section{Sample size}

A similar randomized study (McCracken et al., 2013) using the PHQ-9 to investigate the impact of group-based ACT in a health setting found the ACT group, in comparison with a TAU group, was significantly less depressed at post-treatment $(d=0.46)$ and at 3-month follow-up $(d=0.58)$. Based on these effect sizes, a power analysis was conducted using G* Power (Faul, Erdfelder, Lang, \& Buchner, 2007). In order for sufficient power (.80) and using standard parameters of alpha $=.05$, a total sample size of between 40 and 64 participants was required. 


\section{Statistical methods}

IBM SPSS Statistics 23 (IBM, Released 2014) statistical program was used to analyse the data. An intention-to-treat approach was used with imputation of missing data by last value carried forward (Streiner \& Geddes, 2001). Effect sizes are reported as partial eta-squared (partial $\eta^{2}$ ) and are categorized against the suggested values of .01, .06, and .14 to indicate small, medium, or large effects (Richardson, 2011).

A mixed-design repeated-measure ANOVA was used to analyse the interaction between the two groups and outcome measures across the three time points. Participants from each of the intervention and TAU control groups were combined into a single 'intervention' and 'TAU' group for all statistical analysis. Ideally, a nested design would have been employed. However, the same inclusion and exclusion criteria applied to all groups and the didactic PowerPoint format engendered uniformity across the intervention groups. Given this uniformity, and small number of groups involved, pooling was the preferred strategy. Two mixed-design repeated-measures ANOVAs were conducted to show whether there were significant differences pre-intervention to post-intervention or pre-intervention to 2-month follow-up. To assess group differences in clinically significant change for the primary outcome of depression, a chi-square analysis was conducted.

\section{Ethical approval}

This study was approved by the London - City \& East NHS Research Ethics Committee.

\section{Results}

\section{Participant flow}

Figure 1 illustrates the flow of participants through each arm of the study. Data on those initially invited to the study by the clinical teams could not be recorded as ethical approval allowed details to be collected only after consent. Fifty-three participants were recruited in total, and analysis was completed on all recruited participants in their original assigned groups, ACT $N=26$, and control $N=27$. All 26 participants in the group-based ACT intervention attended at least three of the four sessions, of which 19 (73.1\%) attended all four sessions. The attrition rate was low: 25 (96.2\%) and 22 (84.7\%) participants completed the post-treatment and the 2-month follow-up assessments in the intervention arm and $23(85.2 \%)$ and $25(92.6 \%)$ in the control arm. As a result of the low rate of dropouts, the predictors of dropout were not subjected to further analysis. Total group sizes varied from 6 to 22 attendees across the four groups, of which approximately 40 $50 \%$ were carers who were not eligible to participate in the study. In addition, clinical teams offered the ACT course to a small number of their patients a short time before the groups commenced, which was after study participants had been randomized; thus, recruitment to the study was closed as any further participants could not be randomized. The number of stroke survivors who consented to participant in the research study within each group varied from three to nine (Table 2).

\section{Sample characteristics}

The means and standard deviations of participant demographics and outcome measures at baseline are summarized in Table 3. Independent sample $t$-tests were conducted with Bonferroni correction, to compare the groups at pre-intervention. Significant differences 


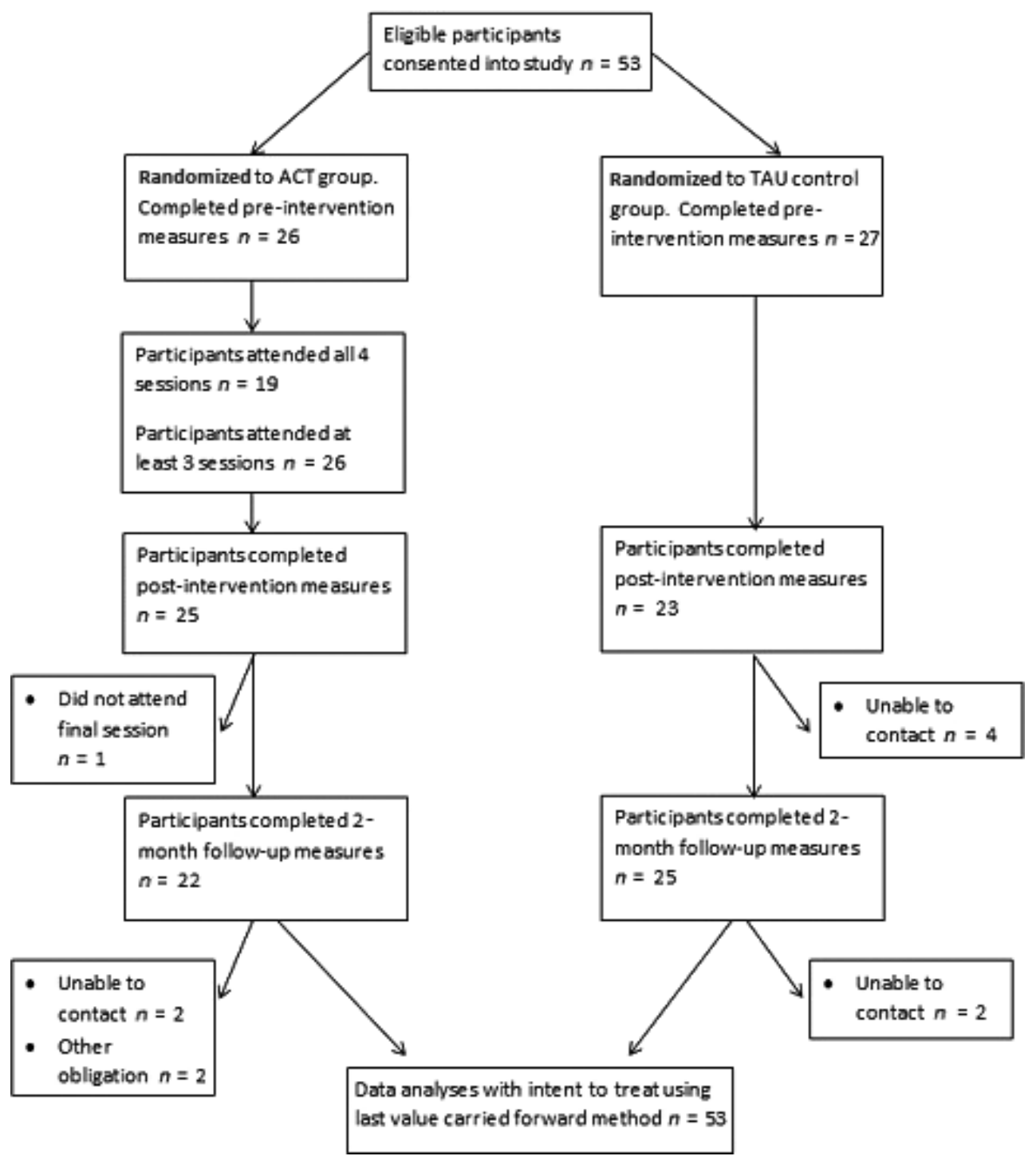

Figure I. Flow chart of participants in randomized groups.

Table 2. Group numbers for the research study

\begin{tabular}{lccccc}
\hline & Group I $(n)$ & Group 2 $(n)$ & Group 3 $(n)$ & Group 4 $(n)$ & Total \\
\hline ACT & 7 & 7 & 3 & 9 & 26 \\
Control & 4 & 6 & 7 & 10 & 27 \\
\hline
\end{tabular}

were found for gender whereby the ACT group had significantly more males. Although qualitative data were collected regarding stroke details (e.g., location and type), the data were too heterogeneous to analyse. There were no significant differences between the groups on the outcome measures at baseline. 
Table 3. Participant characteristics at baseline (Mean [SD] or $n[\%]$ )

\begin{tabular}{|c|c|c|c|c|}
\hline Characteristics & $\mathrm{ACT}(n=26)$ & $\begin{array}{l}\text { Control } \\
(n=27)\end{array}$ & $\begin{array}{l}\text { Overall } \\
(n=53)\end{array}$ & $p$ Value \\
\hline Mean age (years) & $65.3(11.9)$ & $60.0(15.6)$ & $62.7(13.9)$ & .184 \\
\hline Gender (\% male) & $21(80.8)$ & II (40.7) & $32(60.4)$ & $.002 *$ \\
\hline Has had more than one stroke & $6(23.1)$ & $8(29.6)$ & $14(26.4)$ & .538 \\
\hline Months since most recent stroke & I4.I (|4.5) & I3.I (13.3) & I3.6 (I3.7) & .824 \\
\hline Months since first stroke (if had multiple) & $62.5(73.7)$ & $40(37.1)$ & $50(50.8)$ & .380 \\
\hline Age left education & $18.5(3.6)$ & $17.0(2.1)$ & $17.8(3.0)$ & .090 \\
\hline Currently employed & $4(15.4)$ & $10(37)$ & $14(26.4)$ & .05 \\
\hline Currently retired & $19(73.1)$ & $13(48.1)$ & $32(60.4)$ & .124 \\
\hline \multicolumn{5}{|l|}{ Living circumstance } \\
\hline Living with carer & $19(73.1)$ & $5(18.5)$ & $24(45.3)$ & .003 \\
\hline Living with someone who is not carer & $3(11.5)$ & $12(44.4)$ & $15(28.3)$ & \\
\hline Living alone & $4(15.4)$ & $7(25.9)$ & II (20.8) & \\
\hline Not stated & $0(0)$ & $3(11.2)$ & $3(5.6)$ & \\
\hline $\begin{array}{l}\text { Has previously received treatment } \\
\text { for a mental health condition since stroke }\end{array}$ & II (42.3) & $9(33.3)$ & $20(37.7)$ & .653 \\
\hline \multicolumn{5}{|l|}{ Treatment received } \\
\hline Medication & I (3.8) & $2(7.4)$ & $3(5.7)$ & .923 \\
\hline Psychological therapies & $4(15.4)$ & $2(7.4)$ & $6(11.3)$ & \\
\hline Both the above & $3(1 \mathrm{I} .5)$ & I (3.7) & $4(7.5)$ & \\
\hline Not stated & $3(11.5)$ & $4(14.8)$ & $7(13.2)$ & \\
\hline \multicolumn{5}{|l|}{ Study outcome measures at baseline } \\
\hline PHQ-9 & $12.46(6.3)$ & $10.85(7.5)$ & II.65 (6.9) & .402 \\
\hline GAD-7 & $9.77(6.2)$ & $7.85(6.6)$ & $8.79(6.4)$ & .280 \\
\hline EQ-5D-5L & $0.65(0.26)$ & $0.61(0.28)$ & $0.63(0.27)$ & .554 \\
\hline Health (EuroQoL visual analogue) & $59.62(20.5)$ & $55.67(23.8)$ & $57.6(22.1)$ & .521 \\
\hline AHS & $40.77(14.3)$ & $43.37(13.3)$ & $42.09(13.7)$ & .496 \\
\hline WEMWBS & $40.31(10.5)$ & $42.37(12.7)$ & $41.36(11.6)$ & .522 \\
\hline
\end{tabular}

Notes. ACT $=$ acceptance and commitment therapy; AHS $=$ Adult Hope Scale; EQ-5D-5L $=$ Euroquality of life; GAD-7 = Generalized Anxiety Disorder-7; PHQ-9 = Patient Health Questionnaire-9; $S D=$ standard deviation; TAU = treatment as usual; WEMWBS = Warwick and Edinburgh Mental Well-being Scale.

*Significant value after Bonferroni correction (.05/17 = .0029).

\section{Primary outcome}

A mixed-design repeated-measures ANOVA revealed a significant time $\times$ group interaction for depression, in favour of ACT over TAU, $F(2,102)=3.87, p=.024$, with a medium effect size (partial $\eta^{2}=.07$ ). This interaction is shown in Figure 2 , and means and standard deviations are illustrated in Table 4. This group interaction remained significant at pre-treatment to post-treatment analyses $F(1,51)=4.10$, $p=.048$, with a medium effect size (partial $\eta^{2}=.07$ ) and pre-treatment to 2-month follow-up analyses $F(1,51)=5.90, p=.019$, with a medium effect size (partial $\left.\eta^{2}=.10\right)$. A paired $t$-test comparing post-treatment and follow-up scores for the PHQ-9 showed no significant difference which is commensurate with maintenance of gains. 

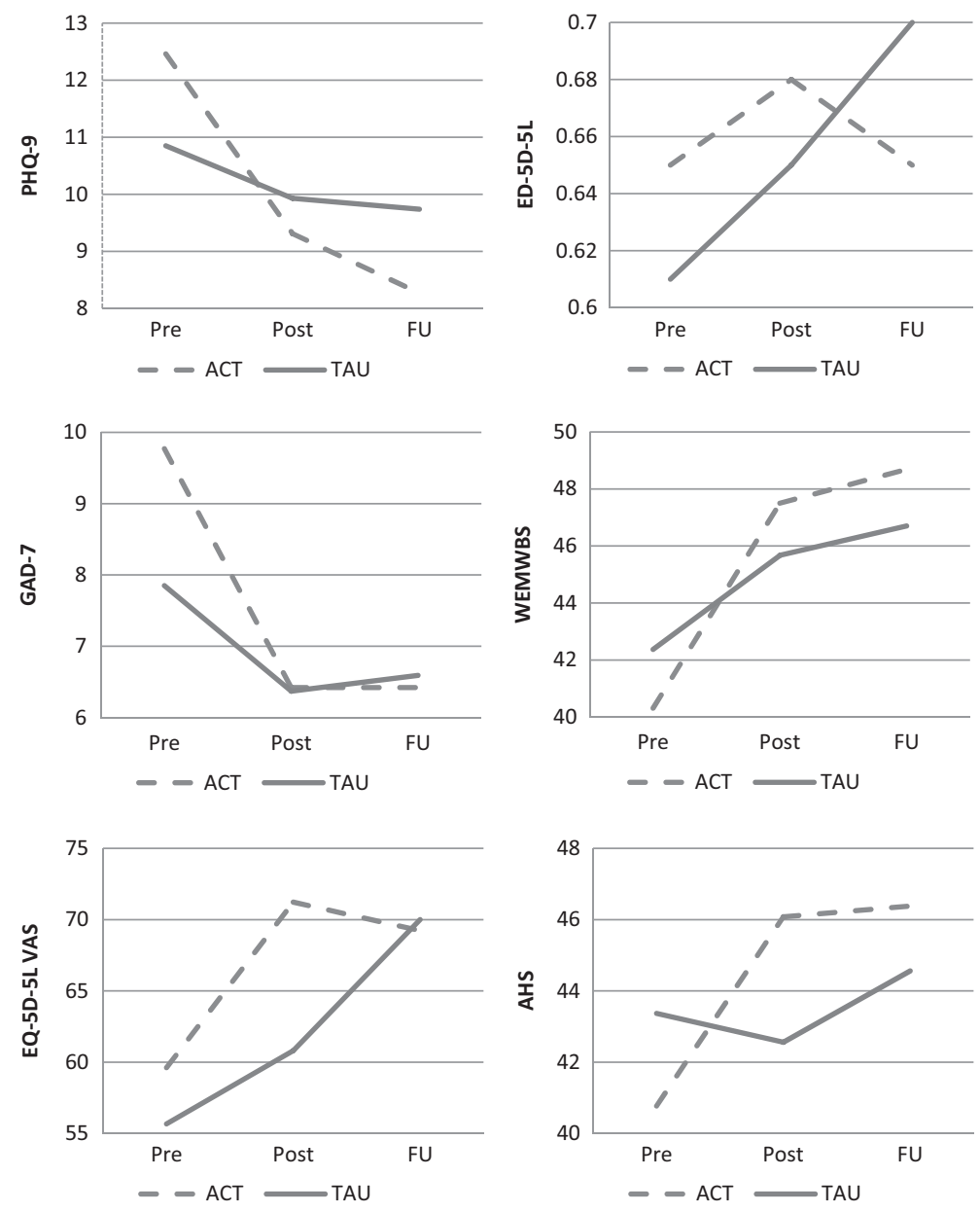

Pre $=$ pre-treatment baseline, Post $=$ post-treatment, $\mathrm{FU}=2$ month follow-up

Figure 2. Line graphs for each outcome measure across groups for the three time points. $\mathrm{FU}=$ 2-month follow-up; Pre = pre-treatment baseline; Post = post-treatment.

\section{Caseness and clinically significant change}

The numbers of cases of depression using the cut-off of 10 or above (Kroenke et al., 2001) were 17 in the intervention group and 15 in the control group at preintervention, 11 and 14 at post-intervention, and 8 and 12 at follow-up. PHQ-9 defines clinically significant change as a score of $\leq 9$ combined with improvement of $50 \%$ from the pre-treatment scores (Kroenke et al., 2001; McMillan, Gilbody, \& Richards, 2010). At post-treatment, a significant group difference occurred, $\chi(1)=5.35, p=.021$, whereby $38.5 \%(N=10)$ of the ACT group had reached a clinically significant change, in comparison with $11.1 \%(N=3)$ in the control group. This significant group difference continued at 2-month follow-up in favour of ACT, $\chi(1)=13.55, p=.001$ : The total number of participants in the ACT group exhibiting clinically significant change from pre-treatment was $53.8 \%(N=14)$, whereas in the control group, it was $7.4 \%(N=2)$. 


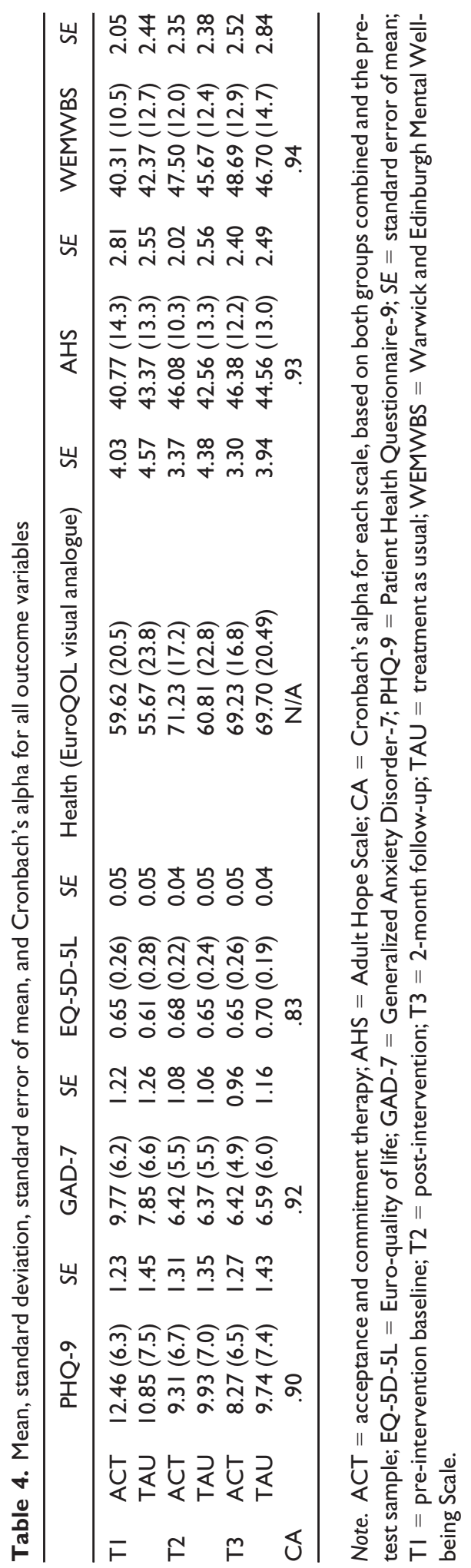




\section{Secondary outcomes}

A mixed-design repeated-measures MANOVA, with the secondary outcome measures across the three phases, revealed a significant overall multivariate main effect for time $\times$ group interaction, Wilks' $\lambda=.56, F(10,42)=3.26, p=.003$, partial $\eta^{2}=.44$. Power to detect the effect was .97. Given the significance of the overall test, the univariate time $\times$ group interactions were examined and significant findings were obtained in favour of ACT over TAU for self-reported health status $F(1.75,102)=4.22, p=.022$, and hopefulness $F(1.65,102)=4.22, p=.017$, all with medium effect sizes (partial $\eta^{2}=.08$ for both variables). No significant effects were found for HRQoL, anxiety, or mental wellbeing. These overall trends can be seen in Figure 2, and overall means and standard deviations, together with Cronbach's alpha for each measure at pre-test, are illustrated in Table 4 .

To assess the outcome measures between the groups and across time points, two mixed-design repeated-measures MANOVAs were conducted to evaluate the interaction from pre-treatment to post-treatment and then from pre-treatment to follow-up. Pretreatment to post-treatment analyses found a significant multivariate effect for the time $\times$ group interaction, Wilks' $\lambda=.70, F(5,47)=4.05, p=.004$, partial $\eta^{2}=.30$. Power to detect the effect was .93 . Univariate main effects revealed significant findings in favour of ACT in comparison with TAU for hopefulness $F(1,51)=10.49, p=.002$, with a large effect size (partial $\eta^{2}=.17$ ). Although mental well-being did not reveal an overall effect across the three time points in the initial repeated MANOVA, pre- to postintervention significance was reached in favour of ACT in comparison with TAU, $F$ (1, $51)=4.16, p=.047$, with a medium effect size (partial $\left.\eta^{2}=.07\right)$. Self-reported health status showed a strong trend in favour of ACT, but did not reach significance $(\phi=.057)$. Significant effects were not found for anxiety or HRQoL in this pre-/post-intervention analysis.

Pre-treatment to follow-up analyses found a significant multivariate effect for time $\times$ group interaction, Wilks' $\lambda=.779, F(5,47)=2.67, p=.033$, partial $\eta^{2}=.22$. Power to detect the effect was .76 . There were no significant univariate effects. Pairedsample $t$-test comparing post-intervention with follow-up scores for all secondary outcome variables showed no significant differences which is consistent with maintenance of gains.

\section{Discussion}

This study found that the ACT intervention significantly reduced depression and increased hopefulness and self-reported health status in stroke survivors in comparison with the TAU control group, with medium effect sizes. However, there were several factors that differed between the ACT and the TAU groups such as being offered a novel treatment, joining a group class, attention, etc. Therefore, it is not possible to attribute the effects to the ACT intervention itself in the absence of additional control measures or the measurement of specific ACT-related processes, such as acceptance. The use of the AAQ (Bond et al., 2011) was considered as a measure acceptance, but a pilot study with stroke survivors suggested that it may be necessary to adapt and revalidate such process measures for the stroke population (Ivey-Williams, 2015).

The participants in the ACT group showed reduction in depression compared with controls from pre-treatment to post-treatment which was maintained during the 2-month follow-up period, with medium effect size. Nearly $54 \%$ of participants in the ACT group 
had had a clinically significant change in depression scores at the 2-month follow-up in comparison with only $7 \%$ of the controls. In addition to the overall interaction, there was an increase in hope scores in favour of ACT from pre-treatment to post-treatment. But this was not maintained at follow-up due to an elevation of the control group's scores. Selfreported health status followed a similar pattern to that of hope. Finally, the outcome variable for mental well-being did not produce overall significant differences across the three time points, but pre-intervention to post-intervention analysis found significant group differences in favour of ACT. Again, the effect was not maintained to follow-up. There were no significant group differences on measures of HRQoL or anxiety. Possibly physical abilities in everyday tasks, such as mobility, self-care, and pain/discomfort, limited scope for increased participation in activities related to quality of life. Alternatively, the 2-month follow-up may not have been sufficient for such life changes to occur in response to the intervention. However, the ACT group did perceived their own health status as significantly better than the control group following the intervention. It is unclear why anxiety showed no significant difference between groups. A systematic review (Swain, Hancock, Hainsworth, \& Bowman, 2013) concluded that there is support for the efficacy of ACT in treating anxiety across a range of populations. Further studies are required to clarify whether post-stroke anxiety can be ameliorated by ACT.

The reduction in depressive symptoms and the increase in self-reported health status and hopefulness in the ACT group are particularly interesting in the context of the aims of ACT, which does not primarily aim to reduce distress or improve health. Instead, ACT aims to help people to live a valued life despite these unpleasant experiences (Harris, 2013). However, the improved hopefulness in the ACT group makes theoretical sense as participants were taught skills to take committed action to move towards a more meaningful life. A conservative interpretation of this result, in line with the ACT philosophy, is that the ACT intervention stimulated participants to experience a change of relationship with their health difficulties as they learn to accept what they cannot change and focus on achievable and meaningful goals. This could also explain the improvements in reports of depressive symptoms which encompass physical and behavioural aspects of function, as well as affect. An alternative explanation is that participants in the control group had a negative reaction to the TAU allocation, and their hopefulness levels decreased at post-intervention, but rose again at the 2-month follow-up, perhaps as a result of being offered the ACT intervention after the study finished.

The significant reduction in depressive symptoms in the ACT group in comparison with the controls mirrors findings of RCTs using group-based ACT with other physical health conditions, such as chronic pain, fibromyalgia, and breast cancer patients (Luciano et al., 2014; McCracken et al., 2013; Mohabbat-Bahar et al., 2015; Wicksell et al., 2013). Compared to TAU or waiting list controls, group-based ACT demonstrated significant improvements on depressive symptoms post-intervention (McCracken et al., 2013; Wicksell et al., 2013; Luciano et al., 2014). These improvements were maintained at 3- or 6-month follow-ups (Luciano et al., 2014; McCracken et al., 2013; Wicksell et al., 2013). However, when group-based ACT was compared to active treatments such as CBT and applied relaxation in chronic pain samples, there were no significant differences between the interventions; all groups reported a reduction in depressive symptoms (Kemani et al., 2015; Wetherell et al., 2011). So the efficacy of ACT in comparison with other treatments in stroke is an area requiring further research.

The increase in hopefulness is consistent with research that found benefits of an ACT intervention with cancer patients (Ghasemi, Dehghan, Farnia, Tatari, \& Alikhani, 2016). It is clinically important as higher levels of hope are associated with improved treatment 
adherence, ability to cope with illness and loss and also enhanced psychological adjustment (Snyder, 2002; Van Servellen, Chang, Garcia, \& Lombardi, 2002; Weis \& Speridakos, 2011).

\section{Implications for service delivery}

In the United Kingdom, many stroke units report no access to psychology services (Royal College of Physicians, 2014); 40\% of stroke survivors felt abandoned after leaving hospital; $50 \%$ did not receive any information or support for anxiety or depression; and two thirds said their emotional needs were not met as well as their physical needs (Stroke Association, 2013). Health care cost for patients with long-term conditions and comorbid depression will typically be $45 \%$ greater than patients without comorbid depression (Naylor et al., 2012). Due to the didactic nature of group-based ACT, it has potential to be a cost-effective low-intensity psychological intervention as, in principle, large numbers of people can attend a course. However, a group setting may be unacceptable for some patients, and therefore, services should offer a range of interventions, tailored to patient need (Royal College of Physicians, 2016).

\section{Strengths and limitations}

The group-based psychoeducational 'ACTivate Your Life after Stoke' intervention is capable of being delivered by non-specialists following suitable training and with support. This ACT intervention was specifically adapted by a team of service users, carers, and professionals from stroke services to ensure the presentation was suitable for the stroke population. The intervention offered stroke survivors access to a community-based psychological intervention, regardless of when their stroke occurred which is in line with guidance (Royal College of Physicians, 2016). This was the first study to explore the outcome of tailored group-based ACT for stroke survivors.

Limitations of this study were its relatively small sample which would not permit more sophisticated statistical analyses without compromising statistical power (e.g., a nested design or analysis of covariance). Despite randomization, the control and intervention samples differed significantly in gender composition and showed a near significant difference for living arrangements. Neither factor is known to reliably influence response to psychological intervention but replication with new, bettermatched samples are indicated. There was no control for use of concomitant treatments and no data for those who declined to participate. The type of participants that are willing to engage in group format psychological interventions, as opposed to individual therapy or medication, may have produced a self-selected sample that biased outcomes (Wylde, Marques, Artz, Blom, \& Gooberman-Hill, 2014). This study was unable to provide equality of therapy hours to compare against an active treatment or undertake independent checks for treatment adherence. However, the intervention was delivered by PowerPoint which facilitated adherence and facilitators received supervision throughout the study.

\section{Conclusions}

This study was designed to evaluate a group-based ACT intervention for stroke survivors, a population for whom few psychological interventions are available. The encouraging results of this current study should be seen as preliminary as the sample size was small in 
comparison with trials intended to produce conclusive results. However, we can conclude that ACT is an acceptable and promising intervention following stroke. Recommendations for further research include a larger sample, with active comparison groups and to assess the cost-effectiveness of this intervention.

\section{Acknowledgements}

We would like to thank Professor Neil Frude for allowing us to adapt his 'ACTivate Your Life' course for stroke and for providing training. We would also like to thank all involved in adapting this ACT course specifically for stroke survivors as well as the study participants.

\section{Funding}

This research did not receive any specific grant from funding agencies in the public, commercial, or not-for-profit sectors.

\section{References}

Adamson, J., Beswick, A., \& Ebrahim, S. (2004). Is stroke the most common cause of disability? Journal of Stroke and Cerebrovascular Diseases: the Official Journal of National Stroke Association, 13(4), 171-177. https://doi.org/10.1016/j.jstrokecerebrovasdis.2004.06.003

Appleby, J., Thompson, J., \& Galea, A. (2012). How is the NHS performing? Quarterly monitoring report September 2012. The King's Fund. Retrieved from https://www.kingsfund.org.uk/ publications/how-nhs-performing-September-2012

Astrom, M. (1996). Generalized Anxiety Disorder in stroke patients. A 3-year longitudinal study. Stroke, 27(2), 270-275. https://doi.org/10.1161/01.STR.27.2.270

Ayerbe, L., Ayis, S. A., Crichton, S., Wolfe, C. D., \& Rudd, A. G. (2014). Natural history, predictors and associated outcomes of anxiety up to 10 years after stroke: The South London Stroke Register. Age and Ageing, 43, 542-547. https://doi.org/10.1093/ageing/aft208

Ayerbe, L., Ayis, S., Wolfe, C. D. A., \& Rudd, A. G. (2013). Natural history, predictors and outcomes of depression after stroke: Systematic review and meta-analysis. British Journal of Psychiatry, 202 (1), 14-21. https://doi.org/10.1192/bjp.bp.111.107664

Bays, C. L. (2001). Quality of life of stroke survivors: A research synthesis. Journal of Neuroscience Nursing, 33, 310-316. https://doi.org/10.1097/01376517-200112000-00005

Bond, F. W., Hayes, S. C., Baer, R. A., Carpenter, K. M., Guenole, N., Orcutt, H. K., . . Zettle, R. D. (2011). Preliminary psychometric properties of the Acceptance and Action Questionnaire-II: A revised measure of psychological inflexibility and experiential avoidance. Behavior Therapy, 42(4), 676-688. https://doi.org/10.1016/j.beth.2011.03.007

Cartwright, J., \& Hooper, N. (2017). Evaluating a transdiagnostic acceptance and commitment therapy psychoeducation intervention. The Cognitive Behaviour Therapist, 10(e9), 1-6. https://doi.org/10.1017/S1754470X17000125

Chung, C. S., Pollock, A., Campbell, T., Durward, B. R., \& Hagen, S. (2013). Cognitive rehabilitation for executive dysfunction in adults with stroke or other adult non-progressive acquired brain damage. Cochrane Database Systematic Review, (4), Cd008391. https://doi.org/10.1002/ 14651858.cd008391.pub2

Department of Health. (2012). Long-term conditions compendium of Information (3rd ed.). Leeds, UK: Author.

Devlin, N., Shah, K. K., Feng, Y., Mulhern, B., \& van Hout, B. (2018). Valuing health-related quality of life: An EQ-5D-5L value set for England. Health Economics, 27(1), 7-22. https://doi.org/10.1002/ hec. 3564 
Dorman, P. J., Waddell, F., Slattery, J., Dennis, M., \& Sandercock, P. (1997). Is the EuroQol a valid measure of health-related quality of life after stroke? Stroke, 28, 1876. https://doi.org/10.1161/ 01.STR.28.10.1876

Faul, F., Erdfelder, E., Lang, A.-G., \& Buchner, A. (2007). G*Power 3: A flexible statistical power analysis program for the social, behavioral, and biomedical sciences. Behavior Research Methods, 39(2), 175-191. https://doi.org/10.3758/BF03193146

Feigin, V. L., Forouzanfar, M. H., Krishnamurthi, R., Mensah, G. A., Connor, M., Bennett, D. A., .. . Murray, C. (2014). Global and regional burden of stroke during 1990-2010: Findings from the Global Burden of Disease Study 2010. Lancet, 383(9913), 245-254. https://doi.org/10.1016/ S0140-6736(13)61953-4

Ghasemi, F., Dehghan, F., Farnia, V., Tatari, F., \& Alikhani, M. (2016). Effectiveness of acceptance and commitment therapy on life expectancy of female cancer patients at Tehran's Dehshpour Institute in 2015. Asian Pacific Journal of Cancer Prevention, 17(8), 4113-4116.

Ghose, S. S., Williams, L. S., \& Swindle, R. W. (2005). Depression and other mental health diagnoses after stroke increase inpatient and outpatient medical utilization three years poststroke. Medical Care, 43(12), 1259-1264. https://doi.org/10.1097/01.mlr.0000185711.50480.13

Gillen, R., Tennen, H., McKee, T. E., \& Gernert-Dott, P. (2001). Depressive symptoms and history of depression predict rehabilitation efficiency in stroke patients. Archives of Physical Medicine and Rehabilitation, 82(1), 1645-1649. https://doi.org/10.1053/apmr.2001.26249

Gillespie, D. C., Bowen, A., Chung, C. S., Cockburn, J., Knapp, P., \& Pollock, A. (2015). Rehabilitation for post-stroke cognitive impairment: An overview of recommendations arising from systematic reviews of current evidence. Clinical Rehabilitation, 29(2), 120-128. https:// doi.org/10.1177/0269215514538982

Godwin, K. M., Ostwald, S. K., Cron, S. G., \& Wasserman, J. (2013). Long-term health related quality of life of survivors of stroke and their spousal caregivers. The Journal of Neuroscience Nursing: Journal of the American Association of Neuroscience Nurses, 45(3), 147-154. https://doi.org/ 10.1097/JNN.0b013e31828a410b

Golicki, D., Niewada, M., Buczek, J., Karlińska, A., Kobayashi, A., Janssen, M. F., \& Pickard, A. S. (2015). Validity of EQ-5D-5L in stroke. Quality of Life Research, 24(4), 845-850. https://doi.org/ 10.1007/s11136-014-0834-1

Graham, C. D., Gillanders, D., Stuart, S., \& Gouick, J. (2014). An acceptance and commitment therapy (ACT)-based intervention for an adult experiencing post-stroke anxiety and medically unexplained symptoms. Clinical Case Studies, 14(2), 83-97. https://doi.org/10.1177/ 1534650114539386

Graham, C. D., Gouick, J., Krahé, C., \& Gillanders, D. (2016). A systematic review of the use of acceptance and commitment therapy (ACT) in chronic disease and long-term conditions. Clinical Psychology Review, 46, 46-58. https://doi.org/10.1016/j.cpr.2016.04.009

Gregg, J. A., Callaghan, G. M., Hayes, S. C., \& Glenn-Lawson, J. L. (2007). Improving diabetes selfmanagement through acceptance, mindfulness, and values: A randomized controlled trial. Journal of Consulting and Clinical Psychology, 75(2), 336-343. https://doi.org/10.1037/0022006X.75.2.336

Hackett, M. L., Anderson, C. S., House, A., \& Halteh, C. (2008). Interventions for preventing depression after stroke. Cochrane Database Systematic Review, (3), Cd003689. https://doi. org/10.1002/14651858.cd003689.pub3

Hackett, M. L., Anderson, C. S., House, A., \& Xia, J. (2008). Interventions for treating depression after stroke. Cochrane Database Systematic Review, (4), Cd003437. https://doi.org/10.1002/ 14651858.cd003437.pub3

Hackett, M. L., \& Pickles, K. (2014). Part I: Frequency of depression after stroke: An updated systematic review and meta-analysis of observational studies. International Journal of Stroke, 9(8), 1017-1025. https://doi.org/10.1111/ijs.12357

Hackett, M. L., Yapa, C., Parag, V., \& Anderson, C. S. (2005). Frequency of depression after stroke: A systematic review of observational studies. Stroke, 36, 1330-1340. https://doi.org/10.1161/ 01.str.0000165928.19135.35 
Harris, R. (2013). Getting unstuck in ACT: A clinician's guide to overcoming common obstacles in acceptance and commitment therapy. Oakland, CA: New Harbinger.

Hayes, S. C., Strosahl, K. D., \& Wilson, K. G. (1999). Acceptance and commitment therapy: An experiential approach to behavior change. New York, NY: Guilford Press.

Herdman, M., Gudex, C., Lloyd, A., Janssen, M. F., Kind, P., \& Parkin, D. (2011). Development and preliminary testing of the new five-level version of EQ-5D (EQ-5D-5L). Quality of Life Research, 20(10), 1727-1736. https://doi.org/10.1007/s11136-011-9903-x

Humphreys, I., Thomas, S., Phillips, C., \& Lincoln, N. (2014). Cost analysis of the Communication and Low Mood (CALM) randomised trial of behavioural therapy for stroke patients with aphasia. Clinical Rebabilitation, 29(1), 30-41. https://doi.org/10.1177/ 0269215514537656

Hunsley, J. (2002). The cost-effectiveness of psychological interventions. Ottawa, ON: Canadian Psychological Association.

IBM. (Released 2014). IBM SPSS statistics for windows, version 23.0. Armonk, NY: Author.

Ivey-Williams, J. (2015). ACTion after stroke: Exploring the effects of an acceptance and commitment therapy group for adult stroke survivors and carers. Cardiff University. Retrieved from http://orca.cf.ac.uk/76359/

Janssen, M. F., Birnie, E., Haagsma, J. A., \& Bonsel, G. J. (2008). Comparing the standard EQ-5D threelevel system with a five-level version. Value in Health, 11(2), 275-284. https://doi.org/10.1111/ j.1524-4733.2007.00230.x

Kangas, M., \& McDonald, S. (2011). Is it time to act? The potential of acceptance and commitment therapy for psychological problems following acquired brain injury. Neuropsychological Rebabilitation, 21(2), 250-276. https://doi.org/10.1080/09602011.2010.540920

Kemani, M. K., Olsson, G. L., Lekander, M., Hesser, H., Andersson, E., \& Wicksell, R. K. (2015). Efficacy and cost-effectiveness of acceptance and commitment therapy and applied relaxation for longstanding pain: A randomized controlled trial. The Clinical Journal of Pain, 31(11), 1004-1016. https://doi.org/10.1097/AJP.0000000000000203

Knapp, P., Campbell Burton, C. A., Holmes, J., Murray, J., Gillespie, D., Lightbody, C. E., . . Lewis, S. R. (2017). Interventions for treating anxiety after stroke. Cochrane Database Systematic Review, 5, Cd008860. https://doi.org/10.1002/14651858.cd008860.pub3

Kroenke, K., Spitzer, R. L., \& Williams, J. B. (2001). The PHQ-9: Validity of a brief depression severity measure. Journal of General Internal Medicine, 16(9), 606-613. https://doi.org/10.1046/j. 1525-1497.2001.016009606.x

Kroenke, K., Spitzer, R. L., Williams, J. B., Monahan, P. O., \& Lowe, B. (2007). Anxiety disorders in primary care: Prevalence, impairment, comorbidity, and detection. Annals of Internal Medicine, 146(5), 317-325. https://doi.org/10.7326/0003-4819-146-5-20070306000004

Lang, A. J., Schnurr, P. P., Jain, S., Raman, R., Walser, R., Bolton, E., . . Benedek, D. (2012). Evaluating transdiagnostic treatment for distress and impairment in veterans: A multi-site randomized controlled trial of acceptance and commitment therapy. Contemporary Clinical Trials, 33(1), 116-123. https://doi.org/10.1016/j.cct.2011.08.007

Lapadatu, I., \& Morris, R. (2017). The relationship between stroke survivors' perceived identity and mood, self-esteem and quality of life. Neuropsychological Rehabilitation, 1-15. https://doi.org/ $10.1080 / 09602011.2016 .1272468$

Lerdal, A., Bakken, L. N., Rasmussen, E. F., Beiermann, C., Ryen, S., Pynten, S., . . Kim, H. S. (2011). Physical impairment, depressive symptoms and pre-stroke fatigue are related to fatigue in the acute phase after stroke. Disability and Rehabilitation, 33(4), 334-342. https://doi.org/10. 3109/09638288.2010.490867

Lincoln, N., \& Flannaghan, T. (2003). Cognitive behavioral psychotherapy for depression following stroke: A randomized controlled trial. Stroke, 34(1), 111-115. https://doi.org/10.1161/01.STR. 0000044167.44670 .55

Lincoln, N. B., Kneebone, I. I., Macniven, J. A. B., \& Morris, R. (2012). Psychological management of stroke. Oxford, UK: Wiley-Blackwell. 
Loetscher, T., \& Lincoln, N. B. (2013). Cognitive rehabilitation for attention deficits following stroke. Cochrane Database of Systematic Reviews, (5), CD002842. https://doi.org/10.1002/146518 58.CD002842.pub2

Luciano, J. V., Guallar, J. A., Aguado, J., Lopez-Del-Hoyo, Y., Olivan, B., Magallon, R., ... GarciaCampayo, J. (2014). Effectiveness of group acceptance and commitment therapy for fibromyalgia: A 6-month randomized controlled trial (EFFIGACT study). Pain, 155(4), 693702. https://doi.org/10.1016/j.pain.2013.12.029

Lundgren, T., Dahl, J., Melin, L., \& Kies, B. (2006). Evaluation of acceptance and commitment therapy for drug refractory epilepsy: A randomized controlled trial in South Africa-A pilot study. Epilepsia, 47(12), 2173-2179. https://doi.org/10.1111/j.1528-1167.2006.00892.x

Lundgren, T., Dahl, J., Yardi, N., \& Melin, L. (2008). Acceptance and commitment therapy and yoga for drug-refractory epilepsy: A randomized controlled trial. Epilepsy \& Behavior: E\&B, 13(1), 102-108. https://doi.org/10.1016/j.yebeh.2008.02.009

McCracken, L. M., Sato, A., \& Taylor, G. J. (2013). A trial of a brief group-based form of acceptance and commitment therapy (ACT) for chronic pain in general practice: Pilot outcome and process results. The Journal of Pain, 14(11), 1398-1406. https://doi.org/10.1016/j.jpain.2013.06.011

McKevitt, C., Fudge, N., Redfern, J., Sheldenkar, A., Crichton, S., Rudd, A. R., . . Wolfe, C. D. (2011). Self-reported long-term needs after stroke. Stroke, 42(2), 1398-1403. https://doi.org/10.1161/ strokeaha.110.598839

McMillan, D., Gilbody, S., \& Richards, D. (2010). Defining successful treatment outcome in depression using the PHQ-9: A comparison of methods. Journal of Affective Disorders, 127(13), 122-129. https://doi.org/10.1016/j.jad.2010.04.030

Mohabbat-Bahar, S., Maleki-Rizi, F., Akbari, M. E., \& Moradi-Joo, M. (2015). Effectiveness of group training based on acceptance and commitment therapy on anxiety and depression of women with breast cancer. Iranian Journal of Cancer Prevention, 8(2), 71-76.

Naylor, C., Parsonage, M., McDaid, D., Knapp, M., Fossey, M., \& Galea, A. (2012). Long-term conditions and mental bealth: The cost of co-morbidities. London, UK: The King's Fund.

Nordin, L., \& Rorsman, I. (2012). Cognitive behavioural therapy in multiple sclerosis: A randomized controlled pilot study of acceptance and commitment therapy. Journal of Rebabilitation Medicine, 44(1), 87-90. https://doi.org/10.2340/16501977-0898

Ost., L. G. (2014). The efficacy of acceptance and commitment therapy: An updated systematic review and meta-analysis. Behaviour Research and Therapy, 61, 105-121. https://doi.org/10. 1016/j.brat.2014.07.018

Plant, S. E., Tyson, S. F., Kirk, S., \& Parsons, J. (2016). What are the barriers and facilitators to goalsetting during rehabilitation for stroke and other acquired brain injuries? A systematic review and meta-synthesis. Clinical Rehabilitation, 30(9), 921-930. https://doi.org/10.1177/026921551 6655856

Richardson, J. T. E. (2011). Eta squared and partial eta squared as measures of effect size in educational research. Educational Research Review, 6(2), 135-147. https://doi.org/10.1016/ j.edurev.2010.12.001

Royal College of Physicians. (2014). Sentinel Stroke National Audit Programme (SSNAP): Acute organisational audit report. London, UK: Royal College of Physicians, Clinical Effectiveness and Evaluation Unit on behalf of the Intercollegiate Stroke Working Party.

Royal College of Physicians. (2015). Sentinel Stroke National Audit Programme (SSNAP): Clinical audit April-June 2015 public report. London, UK: Royal College of Physicians, Clinical Effectiveness and Evaluation Unit on behalf of the Intercollegiate Stroke Working Party.

Royal College of Physicians. (2016). National clinical guideline for stroke: Prepared by the Intercollegiate Stroke Working Party. London, UK: Royal College of Physicians, Clinical Effectiveness and Evaluation Unit on behalf of the Intercollegiate Stroke Working Party.

Ruiz, F. J. (2012). Acceptance and commitment therapy versus traditional cognitive behavioral therapy: A systematic review and meta-analysis of current empirical evidence. International Journal of Psychology \& Psychological Therapy, 12(3), 333-357. 
Snyder, C. R. (2002). TARGET ARTICLE: Hope theory: Rainbows in the mind. Psychological Inquiry, 13(4), 249-275. https://doi.org/10.1207/S15327965PLI1304_01

Snyder, C. R., Harris, C., Anderson, J. R., Holleran, S. A., Irving, L. M., Sigmon, S. T., . . Harney, P. (1991). The will and the ways: Development and validation of an individual-differences measure of hope. Journal of Personality and Social Psychology, 60(4), 570-585. https://doi.org/10. 1037/0022-3514.60.4.570

Soo, C., Tate, R. L., \& Lane-Brown, A. (2011). A systematic review of acceptance and commitment therapy (ACT) for managing anxiety: Applicability for people with acquired brain injury? Brain Impairment, 12(1), 54-70. https://doi.org/10.1375/brim.12.1.54

Spitzer, R. L., Kroenke, K., Williams, J. B., \& Lowe, B. (2006). A brief measure for assessing Generalized Anxiety Disorder: The GAD-7. Archives of Internal Medicine, 166(10), 1092-1097. https://doi.org/10.1001/archinte.166.10.1092

Streiner, D., \& Geddes, J. (2001). Intention to treat analysis in clinical trials when there are missing data. Evidence Based Mental Health, 4(3), 70. https://doi.org/10.1136/ebmh.4.3.70

Stroke Association. (2013). Feeling overwhelmed: The emotional impact of stroke. London, UK: Author.

Stroke Association. (2016). State of the nation: Stroke statistics January 2016. London, UK: Author. Sturm, J. W., Donnan, G. A., Dewey, H. M., Macdonell, R. A., Gilligan, A. K., Srikanth, V., \& Thrift, A. G. (2004). Quality of life after stroke: The North East Melbourne Stroke Incidence Study (NEMESIS). Stroke, 35(10), 2340-2345. https://doi.org/10.1161/01.STR.0000141977.18520.3b

Sugavanam, T., Mead, G., Bulley, C., Donaghy, M., \& van Wijck, F. (2013). The effects and experiences of goal setting in stroke rehabilitation - A systematic review. Disability and Rehabilitation, 35(3), 177-190. https://doi.org/10.3109/09638288.2012.690501

Swain, J., Hancock, K., Dixon, A., Koo, S., \& Bowman, J. (2013). Acceptance and commitment therapy for anxious children and adolescents: Study protocol for a randomized controlled trial. Trials, 14, 140. https://doi.org/10.1186/1745-6215-14-140

Swain, J., Hancock, K., Hainsworth, C., \& Bowman, J. (2013). Acceptance and commitment therapy in the treatment of anxiety: A systematic review. Clinical Psychology Review, 33(8), 965-978. https://doi.org/10.1016/j.cpr.2013.07.002

Tennant, R., Hiller, L., Fishwick, R., Platt, S., Joseph, S., Weich, S., . . Stewart-Brown, S. (2007). The Warwick-Edinburgh Mental Well-being Scale (WEMWBS): Development and UK validation. Health and Quality of Life Outcomes, 5, 63. https://doi.org/10.1186/1477-7525-5-63

Thomas, S. A., Walker, M. F., Macniven, J. A., Haworth, H., \& Lincoln, N. B. (2013). Communication and Low Mood (CALM): A randomized controlled trial of behavioural therapy for stroke patients with aphasia. Clinical Rehabilitation, 27(5), 398-408. https://doi.org/10.1177/026921551 2462227

Townend, E., Tinson, D., Kwan, J., \& Sharpe, M. (2010). 'Feeling sad and useless': An investigation into personal acceptance of disability and its association with depression following stroke. Clinical Rebabilitation, 24(6), 555-564. https://doi.org/10.1177/0269215509358934

van Eeden, M., van Mastrigt, G. A. P. G., Evers, S. M. A. A., van Raak, E. P. M., Driessen, G. A. M., \& van Heugten, C. M. (2016). The economic impact of mental healthcare consumption before and after stroke in a cohort of stroke patients in the Netherlands: A record linkage study. BMC Health Services Research, 16(1), 688. https://doi.org/10.1186/s12913-016-1915-3

Van Servellen, G., Chang, B., Garcia, L., \& Lombardi, E. (2002). Individual and system level factors associated with treatment nonadherence in human immunodeficiency virus-infected men and women. AIDS Patient Care and STDs, 16(6), 269-281. https://doi.org/10.1089/1087291026 0066705

Veehof, M. M., Trompetter, H. R., Bohlmeijer, E. T., \& Schreurs, K. M. G. (2016). Acceptanceand mindfulness-based interventions for the treatment of chronic pain: A meta-analytic review. Cognitive Behaviour Therapy, 45(1), 5-31. https://doi.org/10.1080/16506073. 2015.1098724

Visser, M. M., Heijenbrok-Kal, M. H., van't Spijker, A., Lannoo, E., Busschbach, J. J. V., \& Ribbers, G. M. (2015). Problem-solving therapy during outpatient stroke rehabilitation improves coping and 
health-related quality of life. Stroke, 47(1), 135-142. https://doi.org/10.1161/strokeaha.115. 010961

Watkins, C. L., Auton, M. F., Deans, C. F., Dickinson, H. A., Jack, C. I., Lightbody, C. E., , . Leathley, M. J. (2007). Motivational interviewing early after acute stroke: A randomized, controlled trial. Stroke, 38(3), 1004-1009. https://doi.org/10.1161/01.str.0000258114.28006.d7

Watkins, C. L., Wathan, J. V., Leathley, M. J., Auton, M. F., Deans, C. F., \& Dickinson, H. A. (2011). The 12-month effects of early motivational interviewing after acute stroke: A randomized controlled trial. Stroke, 42(7), 1956-1961. https://doi.org/10.1161/STROKEAHA.110.602227

Weis, R., \& Speridakos, E. C. (2011). A meta-analysis of hope enhancement strategies in clinical and community settings. Psychology of Well-Being: Theory, Research and Practice, 1(1), 5. https://doi.org/10.1186/2211-1522-1-5

Wetherell, J. L., Afari, N., Rutledge, T., Sorrell, J. T., Stoddard, J. A., Petkus, A. J., . . Atkinson, J. H. (2011). A randomized, controlled trial of acceptance and commitment therapy and cognitivebehavioral therapy for chronic pain. Pain, 152(9), 2098-2107. https://doi.org/10.1016/j.pain. 2011.05.016

WHO Monica Project Principal Investigators. (1988). The world health organization monica project (monitoring trends and determinants in cardiovascular disease): A major international collaboration. Journal of Clinical Epidemiology, 41(2), 105-114. https://doi.org/10.1016/ 0895-4356(88)90084-4

Wicksell, R. K., Kemani, M., Jensen, K., Kosek, E., Kadetoff, D., Sorjonen, K., . . Olsson, G. L. (2013). Acceptance and commitment therapy for fibromyalgia: A randomized controlled trial. European Journal of Pain (London, England), 17(4), 599-611. https://doi.org/10.1002/j.1532-2149. 2012.00224.x

Williams, L. S., Brizendine, E. J., Plue, L., Bakas, T., Tu, W., Hendrie, H., \& Kroenke, K. (2005). Performance of the PHQ-9 as a screening tool for depression after stroke. Stroke, 36(3), 635638. https://doi.org/10.1161/01.str.0000155688.18207.33

Wu, S., Kutlubaev, M. A., Chun, H. Y., Cowey, E., Pollock, A., \& Macleod, M. R. (2015). Interventions for post-stroke fatigue. Cochrane Database Systematic Review, (7), Cd007030. https://doi.org/ 10.1002/14651858.cd007030.pub3

Wylde, V., Marques, E., Artz, N., Blom, A., \& Gooberman-Hill, R. (2014). Effectiveness and costeffectiveness of a group-based pain self-management intervention for patients undergoing total hip replacement: Feasibility study for a randomized controlled trial. Trials, 15, 176. https://doi. org/10.1186/1745-6215-15-176

Received 17 November 2017 